\title{
Types of leaky SAW degeneracy in crystals
}

\author{
V. G. Mozhaev, F. Bosia*, M. Weihnacht*
}

Faculty of Physics, Moscow State University, 117234 Moscow, Russia

*Institut für Festkörper- und Werkstofforschung, Helmholtzstraße 20, 01069 Dresden, Germany

\begin{abstract}
Various types of leaky surface acoustic wave (SAW) degeneracies in crystals are considered and systematically classified. Concrete examples of these degenerac; types are given. The possibility of existence of subsonic leaky SAWs is numerically predicted. New correct equations for acoustic axes oblique to symmetry planes in trigonal crystals are derived. It is found that the maximum number of acoustic axes in trigonal crystals is equal to 16 rather than 10 as was previously stated in the literature.
\end{abstract}

\section{INTRODUCTION}

Cases of degeneracy of leaky surface acoustic waves (SAWs) in crystals are of interest for two reasons. The first is that a more penetrating insight into the nature and the properties of leaky SAWs can be achieved by studying their behaviour in limiting conditions, as in degeneracy cases. The other more practical reason is that there is the possibility of using degeneracy forms of leaky SAWs to improve the efficiency of the computational search for these waves in a fundamental way. The present paper is an attempt to consider and to systematically classify various types of leaky SAW degeneracies that can arise for arbitrary choices of substrate and crystal orientation. Based on data from the literature and the results of our previous and present studies in this field, a classification scheme for degeneracy forms of leaky SAWs in crystals is suggested. Concrete examples are given for the degeneracy types considered.

\section{LIMITING BULK WAVES}

Leaky SAWs in crystals may include one or two radiated bulk waves, depending on the value of their phase velocities with respect to those of bulk waves. Since the imaginary part of the wave vector of leaky SAWs, as a rule, is much smaller than its real part, a clear pictorial estimation of the number of radiated bulk waves and their propagation directions can be obtained by considering bulk wave slowness curves in the sagittal plane. As an example, such slowness curves are shown in Fig. 1 for $\mathrm{Ca}(\mathrm{HCOO})_{2}$ (calcium formate). The figure refers to the sagittal plane of the $Y$-cut of the crystal when the propagation direction deviates by $5^{\circ}$ from the crystallographic $X$ axis. The vertical axis in the figure is normal to the surface. Intersections between vertical lines (corresponding to different values of phase velocity of the leaky SAW) and slowness curves determine possible propagation directions of bulk waves. The number of intersections in different intervals of the slowness range ue indicated. The number of radiated bulk waves is equal to half the number of the intersections because the other intersections give bulk-wave power flow directed to the surface rather than to the bulk. Leaky SAWs with these properties may be realized in a layered structure, with the piezoelectric or mechanical coupling of a lower fast substrate and an upper slow crystalline substrate via an air gap. Small coupling of the substrates and an arbitrary choice of the lower substrate provide the possibility to obtain a leaky SAW velocity in any one of the slowness intervals indicated in Fig. 1. One can see that the number of radiated bulk waves in the leaky SAW is not a monotone discrete function of phase velocity and so this number is not completely determined by the wellknown terms "fast" and "slow" leaky waves. The velocity intervals with different numbers of radiated bulk waves corresponding to Fig. 1 are separated by special values of phase velocities referred to as limiting velocities.

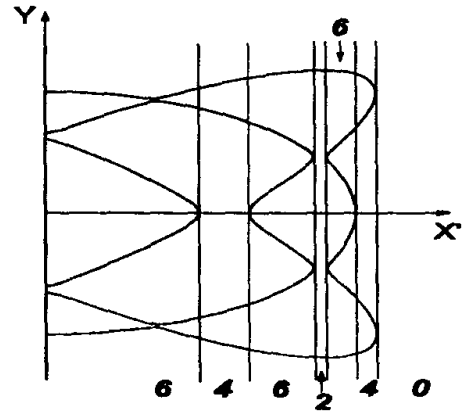

Fig. 1. Cross-section of slowness surface of bulk acoustic waves in $\mathrm{Ca}(\mathrm{HCOO})_{2}$ in the $X Y$ plane obtained through the rotation of the $X Y$ plane by $5^{\circ}$ around the $Y$ axis.

It is instructive, following Viktorov [1], to consider also the isotropic case. The solutions of the bicubic secular equation of Rayleigh waves as a function of Poisson's ratio of an isotropic medium, $\sigma$, is given in Fig. 2. The low-velocity solution corresponding to the Rayleigh wave is real in all cases. Two other highvelocity solutions are real at $\sigma<\sigma_{0}$, where $\sigma_{a} \approx 0.26$. In this case these two solutions describe the Brewsterangle reflections of homogeneous (along the phase 
front) bulk acoustic waves. At $\sigma>\sigma_{0}$ high-velocity solutions become complex: they have the same real part and are equal in value but opposite in the sign of the imaginary parts. Viktorov considered one of these to be a leaky wave. To correctly interpret this solution it is useful to compare it with the limiting velocity.

Prior to this comparison, let us first discuss the difference between leaky SAWs and bulk waves reflected at the surface. A leaky SAW is a superposition of radiated bulk waves and surface waves. In the absence of leakage, the time-average power flow of a surface wave is directed along the surface, that is, its component normal to the surface is equal to zero. In a leaky wave, the power flows of surface partial waves are tilted to the surface to compensate, at the surface, the normal component of the power flow of the radiated bulk waves. However, if the attenuation of the leaky SAW along the surface tends to zero, the normal components of the power flows of surface partial waves also necessarily tend to zero. By contrast, the normal component of the power flow of bulk waves which are incident to the surface is not zero, even when the decaying of the wave field along the surface tends to zero (this corresponds to the transition from incident bulk waves which are inhomogeneous along the phase front to homogeneous ones). A limiting velocity separates these two types of behaviour since the power flow for limiting bulk waves is directed strictly along the surface and its normal component is zero. Thus, the solution under discussion, which includes partial waves which are decaying and increasing into the depth, should be considered more as a solution of a Brewster-angle reflection problem than as a true leaky SAW if its phase velocity along the surface is greater than the fastest limiting velocity. This conclusion is in agreement with the results of Ref. [2].

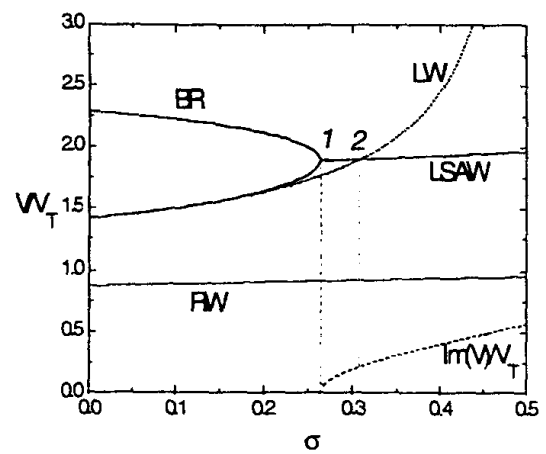

Fig. 2. Solutions of the bicubic secular equation for Rayleigh waves ( $R W)$ in isotropic solids. Left of point $I$ are Brewster reflections (BR) for homogeneous bulk waves, right of point 2 the leaky SAWs (LSAW). LW is the longitudinal wave velocit:
Using this criterion and turning back to Fig. 2, we see that the complex solutions of the Rayleigh equation in the region between points 1 and 2 are solutions of bulk-wave-reflection type for inhomogeneous incident waves rather than leaky SAWs, because their phase velocity along the surface is greater than the longitudinal wave velocity, which is the limiting velocity in this case. The solution under discussion becomes a true leaky SAW only in the region to the right of point 2 , where very high attenuation occurs.

It is reasonable to assume that in analogous figures for crystals, due to anisotropy, point 1 , of zero attenuation along the surface, and point 2 , of transformation of a reflection-problem solution into a leaky SAW, can approach sufficiently for the attenuation of the leaky SAW at point 2 to be quite small.

\section{NON-ATTENUATING SAWS ON LEAKY WAVE BRANCHES}

The most well-known case of degeneracy of leaky SAWs is probably the case of the basal-cut of cubic crystals like copper, for propagation along the face diagonal of the cube [3-5]. The azimuthal angle $\phi$ measured from one of the crystallographic axes lying in the boundary plane is equal to $45^{\circ}$ for this direction. At $\phi=45^{\circ}$ the attenuation tends to zero and the leaky SAW degenerates into a non-radiating Rayleigh wave polarized in the sagittal plane. For such a type of degeneracy it is necessary that (i) the sagittal plane be a symmetry plane of the crystal, (ii) the Rayleigh wave velocity be greater than the velocity of the limiting shear-horizontal bulk wave. The first condition implies that the orientations for the occurrence of this type of degeneracy, if it exists, do not depend on specific values of elastic constants. In addition to such a symmetrical degeneracy direction, one can find nonsymmetrical degeneracy directions which have no relation to symmetry planes[3-5]. Leaky SAWs in this case degenerate into nonattenuating normal SAWs with mixed polarization. As an example of both these degeneracies, we consider the crystal of gallium nitride (GaN). This hexagonal crystal is a promising material for blue light emitting devices but besides it is piezoelectric, thus becoming a possible candidate for ultrasonic applications. A simple estimation of Rayleigh and $\mathrm{SH}$ bulk wave velocities, $\nu_{R}$ and $\nu_{S H}$, for the $X$ propagation direction on $Y$-cut $\mathrm{GaN}$ using the data for elastic constants from Ref. [6], shows that $v_{R}>v_{S H}$. This is an indication of the possibility of existence of leaky SAWs on this cut. The results of the corresponding calculations are given in Fig. 3. Piezoelectricity is ignored because of the absence of a full set of piezoelectric constants. It is interesting to 
note that as well as the symmetrical leaky-SAW degeneracy direction at $\phi=0^{\circ}$, there are two nonsymmetrical directions, at $\phi \approx 31^{\circ}$ and $\phi \approx 61^{\circ}$, as opposed to only one in the case of $X$-cut quartz [3]. The other interesting peculiarity occurs at the border of the existence region of this leaky wave branch (see section V).

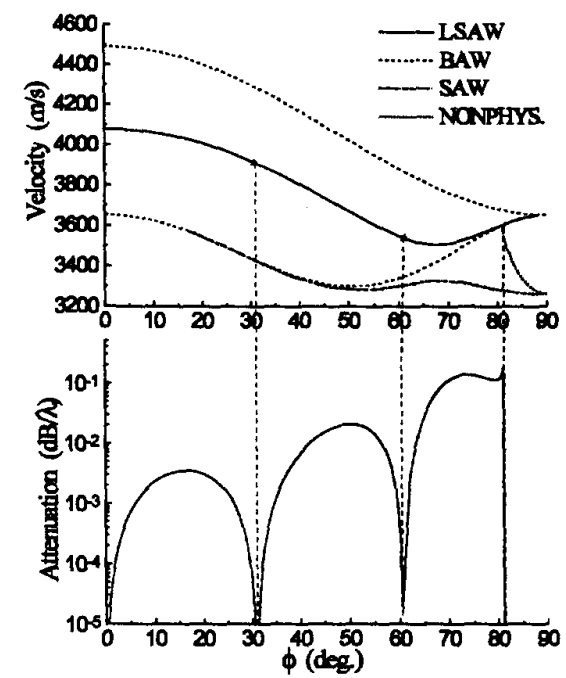

Fig. 3. Phase velocity and attenuation of leaky SAWs (LSAW) on Y-cut GaN as a function of azimuthal angle $\phi$. Velocity curves for bulk acoustic waves (BAW), surface acoustic waves (SAW), and nonphysical solutions (NONPHYS.) are also given.

\section{EXCEPTIONAL-WAVE DEGENERACY}

(a) $S H$-waves propagating along the surface

In some special cases a bulk wave propagating along the surface can satisfy by itself the traction-free boundary conditions. Waves of this type are referred to as exceptional waves. They are one of the types of degeneracy forms of leaky SAWs. The application of exceptional waves to search for leaky SAWs in crystals has been studied by Naumenko [7]. To illustrate this method, we first consider a simple geometry when the propagation direction (the $X$ axis) is the normal to a symmetry plane of the crystal. The stiffness matrix $c_{l J}$ in this case has some zero elements for subscript values of $I=1,2,3,4 ; J=4,5$ Rotation of the coordinate system around the $X$ axis does not change the general form of this matrix. The elastic constant $c$ 's relative to the rotated axes becomes:

$$
c_{56}^{\prime}=\left(\cos ^{2} \theta-\sin ^{2} \theta\right) c_{56}-\cos \theta \sin \theta\left(c_{56}-c_{55}\right),
$$

where $\theta$ is the rotation angle measured from the $Y$ axis. From Eq.(1) it follows that $c^{\prime}{ }_{56}$ is equal to zero at angles given by

$$
\tan 2 \theta=2 c_{56} /\left(c_{66}-c_{55}\right) .
$$

Two pure shear bulk waves can propagate along the $X$ axis of such a crystal with phase velocities given by

$$
\rho v_{1.2}^{2}=\frac{1}{2}\left[\left(c_{55}+c_{66}\right) \pm \sqrt{\left(c_{55}-c_{66}\right)^{2}+4 c_{56}^{2}}\right] \text {. }
$$

It is easy to verify that Eq. (2) determines two orthogonal cuts of a crystal for which one or another of these bulk waves satisfies the free-surface boundary conditions, that is, these waves are exceptional waves with respect to selected cuts. If one chooses the cut of the crystal in such a way that the fast shear wave satisfies the boundary conditions, then one might expect its transformation into a leaky SAW with a small additional rotation of the cut. Eqs. (1) - (3) are applicable to monoclinic and highersymmetry crystals. As a particular case, they include the relations for trigonal crystals given in Ref. [7].

Leaky SAWs propagating along the $X$ axis, on cuts rotated around the same $X$ axis, were found in several trigonal crystals such as lithium niobate [8], lithium titanate [9] and quartz [10]. We have obtained some similar results for gallium orthophosphate, $\mathrm{GaPO}_{4}$ and langasite, $\mathrm{La}_{3} \mathrm{Ga}_{5} \mathrm{SiO}_{14}$. We do not present the corresponding figures because of space limitations. For $\mathrm{GaPO}_{4}$, degeneracies take place at $\phi \approx 75^{\circ}$ into a $\mathrm{SH}$ exceptional wave (if piezoelectricity is ignored), and at $\phi=172.6^{\circ}$ into an isolated solution in the form of a normal non-attenuating SAW with mixed polarization.

(b) $S H$-waves propagating oblique to the surface In the case considered above, the leaky SAW degenerates into a SH bulk wave with wave vector parallel to the surface. In the review by Farnell [3], it is mentioned that the leaky SAW propagating on the (111) plane of copper degenerates into a SH bulk wave with wave vector tilted to the surface, at an angle of $30^{\circ}$ from [110]. Our numerical calculations do not confirm this result. Instead, the leaky SAW degenerates in this case into a sagittally polarized Rayleigh wave which is faster than the $\mathrm{SH}$ exceptional wave. Nevertheless, degeneracy into a SH wave with a tilted wave vector is quite possible. In particular, this type of degeneracy, as we have found using material data from Ref. [11], occurs for rotated $Z$-cut $\mathrm{LiB}_{3} \mathrm{O}_{5}$, in the case when the boundary plane is obtained by a $45^{\circ}$ rotation of the $Z$ plane around the $Y$ axis. Degeneracy into a $S H$ wave with a tilted wave vector occurs at $\phi=0^{\circ}$ from [101].

(c) Waves with mixed polarization

Concerning the case of exceptional waves with mixed polarization, we have not performed any calculations of our own for this type of leaky SAW degeneracy. We remark only that the comparison of results given in Refs. $[7,12]$ for $X$-cut quartz leads us 
to assume that the velocity of the quasishear exceptional wave found for the propagation direction of $41.2^{\circ}$ from the $Y$ axis [7] is probably a degeneracy point on a leaky branch shown in Fig. 3 of Ref. [12].

\section{BULK-WAVE-REFLECTION DEGENERACY}

\section{(a) Brewster-angle reflections}

There are several different cases of degeneracy which can be classified as a degeneracy of bulk-wavereflection type. Among these are Brewster-angle reflections for inhomogeneous or homogeneous waves. First we consider the case of inhomogeneous waves. For this purpose we turn back to the $Y$-cut of GaN (Fig. 3). A highly accurate investigation of the behaviour of leaky waves in the vicinity of the end point of their existence region leads to unexpected results, which are in contradiction with the earlier view of the slowest bulk wave velocity being a lower limit to the value of the leaky SAW velocity. It appears that the leaky SAW attenuation drops continuously, but very sharply, to zero at the termination of leaky wave branch. However, the phase velocity of leaky SAWs in this region is found to be smaller than the phase velocity of the slowest bulk waves. This phenomenon is explained by admitting the leakage of an inhomogeneous bulk wave which may be slower along the surface than homogeneous ones. The end points of the existence regions of these subsonic leaky SAWs correspond to the merging points of two non-physical branches relative to the solutions of Brewster-type reflection problems for inhomogeneous bulk waves.
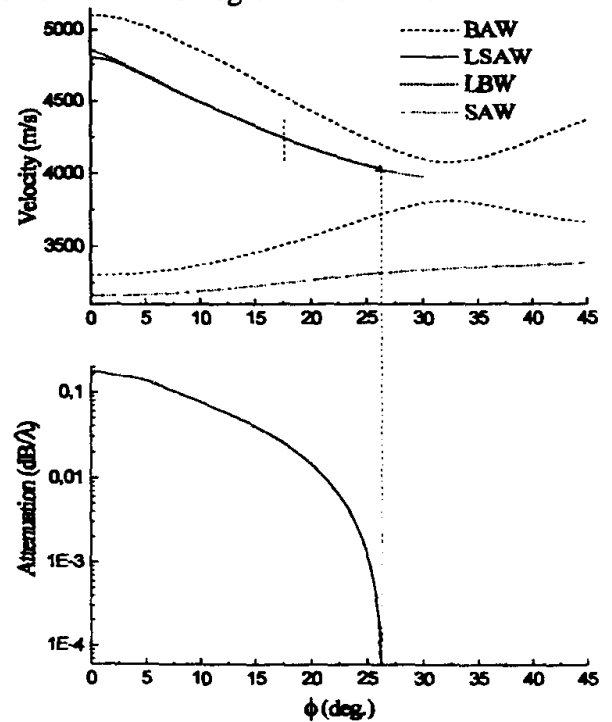

Fig. 4. Phase velocity and attenuation of leaky SAWs on Y-cut quartz $L B W$ indicates the limiting bulk wave.
The next figure (Fig. 4) is relative to $Y$-cut quartz, which is an example of a degeneracy of Brewsterangle-reflection type for homogeneous bulk waves. A leaky SAW branch is found on this cut in the range of angles $-26.28^{\circ}<\phi<26.28^{\circ}$ from the $X$ axis. At the boundary of this range the solution can be identified as a field comprising a homogeneous incident wave and two reflected waves at the Brewster angle of incidence, whereas near $\phi=0^{\circ}$ the solution is undoubtedly a leaky SAW. The change of character in the solution occurs at the propagation direction of $\phi=18.5^{\circ}$ from the $X$ axis indicated by a short dashed line in the figure), where the leaky SAW branch crosses the second limiting bulk wave velocity and becomes faster than it. This situation is somewhat similar to the isotropic case shown in Fig. 2. Analog to Poisson's ratio here is the azimuthal angle.

(b) Waves propagating along acoustic axes

A specific case of degeneracy of bulk-wavereflection type takes place if an acoustic axis is present in the sagittal plane [13]. In this case the degeneracy solution represents the reflection of bulk waves when the incident wave and one reflected wave propagate along the acoustic axis. Such a reflection problem was originally studied in Ref. [14]. Recall that the propagation direction of two acoustic bulk waves dissimilar in polarization is referred to as an acoustic axis of a crystal if their phase velocities coincide. The relationship between leaky SAW branches and acoustic axes has attracted our attention to the problem of finding acoustic-axis directions in crystals. The general theory of acoustic axes in crystals was developed by Khatkevich [15]. He concluded that all acoustic axes in trigonal crystals lie only in symmetry planes, that their number is 4 or 10 , and that previous numerical data for quartz available in the literature [16] are incorrectly calculated. This discrepancy has stimulated us to recalculate Khatkevich's results on the basis of the general equations given in his paper. We have found that the analytical results obtained by Khatkevich for trigonal crystals, and therefore his conclusions mentioned above, are incorrect. The results of our calculations are the following:

$$
\begin{aligned}
& \tan ^{2} \theta=\frac{\left(c_{33}+c_{44}+2 c_{13}\right)\left(c_{11}+c_{12}\right)-2\left(c_{13}+c_{44}\right)^{2}}{\left(c_{11}-c_{44}\right)\left(c_{11}+c_{12}\right)-2 c_{14}{ }^{2}}, \\
& \sin 3 \varphi=\frac{2\left(c_{33}+c_{44}+2 c_{13}\right)-\left(2 c_{11}+c_{13}-c_{44}\right) \tan ^{2} \theta}{c_{14} \tan ^{3} \theta}, \text { (5) }
\end{aligned}
$$

where $\theta$ and $\varphi$ are the polar and azimuthal angles describing the orientation of the acoustic axis, respectively. Eqs. (4) and (5) give real directions oblique to symmetry planes for some trigonal crystals like quartz and berlinite: $\varphi=19.13^{\circ}, \theta=26.96^{\circ}$ for quartz and $\varphi=25.94^{\circ}, \theta=24.54^{\circ}$ for berlinite. These 
values are confirmed by numerical solutions with account of piezoelectricity, which are: $\varphi=19.64^{\circ}, \theta$ $=26.59^{\circ}$ for quartz and $\varphi=25.37^{\circ}, \theta=24.52^{\circ}$ for berlinite. It is of interest to note that according to Eqs. (4) and (5) oblique acoustic axes should also exist in lithium niobate when $\varphi=-21.40^{\circ}, \theta=34.40^{\circ}$. However, numerical calculations of phase velocities of bulk waves in this crystal, taking into account piezoelectricity, reveal small but finite spacing between the two sheets of the slowness surface rather thwn their contact in the corresponding cone of directions. Thus piezoelectricity has a pronounced efiect on the existence of acoustic axes in this special case. On the other hand, directions in lithium niobate determined by Eqs. (4) and (5) may be considered as virtual acoustic axes since a small perturbation of piezoelectric or other material constants is enough to transform virtual acoustic axes into real ones.

Khatkevich's conclusion that all acoustic axes in trigonal crystals lie in symmetry planes has up till now been accepted in literature [17]. The existence of oblique acoustic axes determined by Eqs. (4) and (5) allows us to correct the previous estimation of the number of acoustic axes in trigonal crystals. Taking into account the threefold axis and the symmetry planes, one can conclude that trigonal crystals may additionally have 6 acoustic axes oblique to symmetry planes, that is, the maximum number of acoustic axes is 16 rather than 10 , as was previously believed. An oblique acoustic axis has been used by us to find a leaky SAW branch in quartz. The orientation of the cut and the propagation direction are defined by angles $\left(19.64^{\circ},-26.59^{\circ}, \phi\right)$, with $0^{\circ}<\phi<9.49^{\circ}$, these being three consecutive rotations around the axes $(Z, Y, Z)$.

Two configurations suitable to search for leaky SAWs with the aid of acoustic axes have been established in Ref. [13]. In the first an acoustic axis should be in the plane of the surface. In the other an acoustic axis lying in the sagittal plane is directed into the bulk and one of the bulk waves propagating along it should simultaneously be a limiting wave (erroneously called exceptional wave in Ref. [13]). The interpretation of the degeneracy solution as a solution of a bulk-wave-reflection problem allows us to suggest that all the acoustic-axis orientations confined between the two previously specified directions are permissible. To test this hypothesis, we have investigated some cuts of different crystals like berlinite. langasite, and potassium niobate. In all the cases studied, it is possible to tilt the acoustic axes into the bulk and at the same time retain their connection with a leaky SAW branch. However, the tilt angles, as a rule. are smaller than would be expected from the analysis of slowness curves. These results are conditioned to a large extent by numerical difficulties in calculating solutions with very small attenuation near degeneracy points. The best coincidence between result and prediction is observed in the case of the langasite cut defined by the Euler angles $\left(90^{\circ}, 90^{\circ}, 44.8^{\circ}\right)$. Fig. 5 shows the corresponding slowness curves for the case when the acoustic axis is in the plane of the surface. The angle $\theta=15.21^{\circ}$ between the $Z$ axis and the tangent to the slowness curve in the point of the acoustic axis, which is the maximum expected tilting angle, is marked in Fig. 5. The maximum obtained tiling angle, which enables us to find leaky SAW from the acoustic axis is equal to $14.94^{\circ}$.

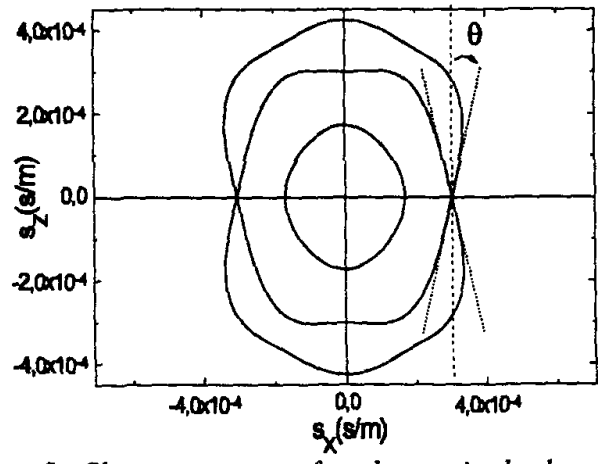

Fig. 5. Slowness curves for the sagittal plane of langasite, Euler angles $\left(90^{\circ}, 90^{\circ}, 44.8^{\circ}\right)$.

(c) Reflection of waves coinciding with limiting bulk waves.

One further variety of degeneracy solution that can be interpreted as a solution of a bulk-wave-reflection problem may occur at intersection points of two different branches of limiting velocities. To study this possibility, we have repeated some well-known calculations of leaky SAW velocity and attenuation on the basal plane of copper $[5,18]$ and on a plane of copper slightly deviated from the basal one [19], with special emphasis on the correlation between specific points of slowness curves and the velocity of the leaky wave branch at its termination. The rotated cut is obtained by a rotation of $1^{\circ}$ of the basal cut (001) around the [110] direction. In the basal cut there is a direct relationship between the leaky SAW degeneracy at $\phi=24.84^{\circ}$ and zero curvature points on the slowness surface. This conclusion is absent in Ref. [18] although figures available there allow one to make this supposition. For the rotated cut, the boundary of the existence region of leaky SAWs defined by the Euler angles $\left(45^{\circ}, 1^{\circ}, 21.44^{\circ}\right)$ corresponds to the coincidence of limiting velocities for convex and concave parts of slowness curves in the sagittal plane (Fig. 6). The same type of degeneracy has been found by Naumenko [20] in quartz. In the general case, such a solution can 
probably include terms that are linearly increasing into the depth as in the Goodier-Bishop solution for the isotropic case [3]. However, this question still remains to be investigated.

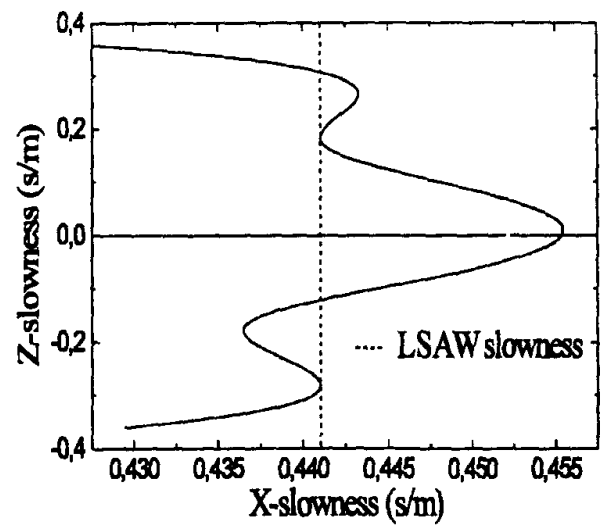

Fig. 6. Quasishear wave slowness curve for the sagittal plane of $\mathrm{Cu}$, Euler angles $\left(45^{\circ}, I^{\circ}, 21.44^{\circ}\right)$.

\section{CONCLUSION}

The following main types of degeneracy forms of leaky SAWs in crystals have been identified:

1. Normal SAWs (without leakage):

(a) Rayleigh waves (SAWs polarized in the sagittal plane),

(b) Isolated solutions with mixed polarization (in the form of three-component, two-partial SAWs in a nonpiezoelectric case).

2. Exceptional bulk waves:

(a) SH-waves propagating along the surface,

(b) SH-waves propagating oblique to the surface,

(c) Waves with mixed polarization.

3. Bulk-wave-reflection solutions with a reduced number of involved partial waves:

(a) Brewster-angle reflections,

(b) Wave propagation along acoustic axes,

(c) Reflection of waves coinciding with limiting bulk waves.

\section{ACKNOWLEDGEMENTS}

This research is supported in part by INTAS (project 96-441), by Sächsiches Ministerium für Kunst und Wissenschaft and by Deutsche Forschungsgemeinschaft. The authors are grateful to Dr. R. Wobst for his programming work.

\section{REFERENCES}

[1] I. A. Victorov, Akust. Zh. 22, 675-678 (1976) [Sov. Phys.-Acoust. 22, 380-382 (1976)].
[2] S. V. Biryukov and M. Weihnacht, J. Appl. Phys. 83, 3276-3287 (1998).

[3] G. W. Farnell, in: Physical Acoustics, Vol. VI, edited by W. P. Mason and R. N. Thurston (Academic Press, New York, 1970), 109-166.

[4] G. W. Farnell, in: Proc. Intern. Symp. Surface Waves in Solids and Layered Structures (ISS. WAS 86)\} Vol. III, (Novosibirsk, USSR, 1986) 7-21.

[5] D. B. Taylor, Proc. R. Soc. Lond. A 376, 265-300 (1981).

[6] A. Polian, M. Grimsditch, and I. Grzegory, J. Appl. Phys. 79, 3343-3344 (1996).

[7] N. F. Naumenko, J. Appl. Phys. 79, 8936-8943 (1996).

[8] K. Yamanouchi and K. Shibayama, J. Appl. Phys. 43, 856-862 (1972).

[9] K. Nakamura, M. Kazumi, and H. Shimizu, in: 1977 IEEE Ultrasonics Symposium Proceedings, (1977), 819-822.

[10] Y. Shimizu and Y. Yamamoto, Electron. Lett. 21, 225-226 (1985).

[11] R. Guo, S. A. Markgraf, Y. Furukawa, M. Sato, and A. S. Bhalla, J. Appl. Phys. 78, 72347239 (1995).

[12] R. M. Taziev, Akust. Zh. 35, 922-928 (1989) [Sov. Phys. Acoust. 35, 535-538, (1989)].

[13] V. G. Mozhaev and M. Weihnacht, in: 1997 IEEE Ultrasonics Symposium Proceedings, (1997) 267-273.

[14] E. N. Koshkina, V. E. Lyamov, and T. A. Mamatova, Kristallografiya 23, 1274-1277 (1978) [Sov. Phys. Crystallogr. 23, 721-723 (1978)].

[15] A. G. Khatkevich, Kristallografiya 7, 742-747 (1963) [Sov. Phys. Crystallograpy 7, 601-604 (1963)].

[16] W. G. Cady, Piezoelectricity (McGraw-Hill Book Company, New Ycrk, 1946).

[17] B. M. Darinskii, Kristallografiya 39, 773-780 (1994) [Crystallography Rep. 39, 697-703 (1994)].

[18] L. Wang and J. Lothe, Wave Motion 16, 101-112 (1992).

[19] L. Wang, in: Statistical Physics Elasticity and Dislocation Theory, Eds. T. Jøssang and D. M. Barnett, Physica Scripta, 1992, vol. T44, 128 132.

[20] N. F. Naumenko, in: 1997 IEEE Ultrasonics Symposium Proceedings, (1997), 255-260. 